\title{
Techniques d'abréviation dans les webchats francophones
}

\author{
Klara Krautgartner (Salzburg)
}

\begin{abstract}
lut toi, asv stp? - In a French speaking webchat you can find a good deal of messages like these which are quite normal for the regular chatter, but often incomprehensible for the non specialist (the example above means 'salut toi, âge, sexe, ville s'il te plait'). Chat communication is characterised by an extreme rapidity. The more users participate, the faster turns disappear from the screen. So the chatters better create very short messages.

This paper intends to study different types of abbreviation through real time written interactions in French speaking webchats. How do users shorten their messages? How do they succeed in communicating a maximum of content using the least possible number of (ASCII-) signs? On the base of examples and statistic data the paper presents conventional types of abbreviation such as $h$ instead of heure, acronyms, apocopes etc. as well as more innovative abbreviation strategies as for instance phonetic writing ( $c$ for $c^{\prime}$ 'est) or words reduced to their consonants (e.g. ms instead of mais). All these strategies constitute an essential linguistic tool in chat writing.
\end{abstract}

\section{Introduction}

La présente étude ${ }^{1}$ vise à une exploration des ressources linguistiques utilisées et inventées par les individus pratiquant les conversations écrites en temps réel sur le web dans le but de raccourcir leurs messages. Ce qui caractérise la plupart des chats, c'est l'extrême rapidité de la communication. Dans un salon (en anglais: chatroom) où le nombre des participants actifs va au-delà de cinq, l'important est que les correspondants comprennent et réagissent vite pour ne pas perdre le fil de la conversation: dans les secondes qui suivent, ils n'auront plus le message sous les yeux. Etant donné cette brièveté de vie des énoncés, la rapidité de rédaction et la concision sont essentielles: "More important in this regard however is the fact that in order to keep up with the flow of conversation it is often necessary to respond quickly and this means that unless one can type very rapidly, messages must be kept short." (Werry 1996: 53). Même dans un salon qui ne comporte que deux ou trois participants, où les messages défilent donc moins rapidement, les chateurs éprouvent le besoin d'être brefs et précis: personne n'aime

\footnotetext{
1 Mes remerciements les plus chaleureux vont à Madame Marianne Kilani-Schoch ainsi qu'à Monsieur JeanMichel Adam, professeurs à l'Université de Lausanne grâce auxquels j'ai pu passer une année d'études fructueuse et riche en expériences en Suisse romande. J'inclus dans ma gratitude les responsables de la Commission fédérale des bourses pour étudiants étrangers pour m'avoir accordée une bourse de la Confédération.
} 
attendre deux minutes pour avoir une réponse de la part de son interlocuteur. L'idée est, dans les deux cas, de dire un maximum en un minimum de temps. C'est pourquoi les chateurs ont recours, pour diminuer le temps de production et de réception, à des stratégies de raccourcissement de nature très diverse. Mon analyse se concentrera sur le niveau morphologique. ${ }^{2}$

\section{Définitions}

J'emploie le terme d'abréviation en son sens large en me référant au Dictionnaire de linguistique et des sciences du langage selon lequel une abréviation est "Toute représentation d'une unité ou d'une suite d'unités par une partie de cette unité ou de cette suite d'unités" (Dubois et al. 1994: 1). Ainsi, je distingue les types suivants:

\subsubsection{Abréviations proprement dites}

Je comprends par "abréviation proprement dite" la réduction d'un mot à quelques lettres seulement de ce mot. Il ne s'agit donc que de créations écrites, la forme abrégée ne se prononce pas. On différencie

l'abréviation par apocope

La réduction affecte la finale du mot, par exemple $M$. (Monsieur), $h$ (heure), chap. (chapitre), bull. (bulletin) etc. On met un point abréviatif à l'exception des unités de mesure $(m, k W h)$ (Encyclopédie Hachette s.a., article abréviation).

\section{l'abréviation par syncope}

L'abréviation s'effectue par la suppression de quelques lettres à l'intérieur du mot, par exemple $d s$ (dans), $b d$ (boulevard), grd (grand) etc. Dans ce cas-là, on ne met pas de point abréviatif.

\subsubsection{Mots tronqués}

Le mot troncation est synonyme de réduction et désigne le fait de créer un nouveau mot en supprimant un ou plusieurs syllabes d'un mot existant (Encyclopédie Hachette s.a., article réduction).

À la différence des abréviations proprement dites, les mots tronqués existent non seulement graphiquement, mais aussi phonétiquement. On distingue

\footnotetext{
${ }^{2}$ Pour ce qui est de l'allemand, ma langue maternelle, il existe un bon nombre d'études à ce sujet, p.ex. Haase et al. 1997, Naumann 1997 (une étude sur l'IRC), Runkehl/Schlobinski/Siever 1998, Storrer 2001, Wilde 2002, Bader 2002 pour ne pas oublier Schlobinski et al. 2001 (ce dernier représente une analyse détaillée des messages écrits envoyés par téléphone portable). Comme les publications concernant les webchats francophones sont beaucoup moins nombreuses (un des rares linguistes à avoir étudié en détail le phénomène est Jacques Anis, professeur à Nanterre et spécialiste du langage sur l'internet, voir bibliographie), j'ai décidé de me limiter à la langue de Molière.
} 
la troncation par apocope

Par analogie à l'abréviation par apocope, c'est la partie finale du mot qui tombe: vélo (vélocipède), ciné (cinéma)...

la troncation par aphérèse

Ce phénomène où l'on supprime la ou les syllabe(s) initiale(s) d'un mot est beaucoup moins fréquent: bus (autobus), stratif (administratif)...

\subsubsection{Sigles}

Un sigle est un mot qui se compose des lettres initiales d'un groupe de mots. La prononciation est soit syllabique, soit alphabétique, soit les deux. Si le sigle est prononcé comme un mot ordinaire (prononciation syllabique), on l'appelle acronyme (Office de la langue française 2002, article acronyme). Ainsi, le terme CERN (Comité Européen de la Recherche Nucléaire) est un acronyme tandis que l'abréviation $T G V$ (train à grande vitesse) qui est prononcée lettre après lettre, est un sigle au sens strict.

\section{$3 \quad$ Présentation des corpus}

Mon étude repose sur treize corpus enregistrés dans trois webchats francophones. Le lecteur intéressé trouvera en annexe une capture d'écran de ces chats. Au total, il s'agit de 3000 messages d'usagers. Afin de faciliter l'analyse, j'ai numéroté les messages en commençant, dans chacun des treize corpus, par le numéro 1. J'attache une grande importance à l'illustration des caractéristiques étudiées à l'aide d'exemples tirés de mes corpus. Les chiffres arabes indiquent le numéro du message cité, les chiffres romains celui du corpus dont il est tiré. L'énoncé suivant par exemple fait partie du corpus III et il y correspond à la ligne 41:

III 41 Sims $\rightarrow$ Salut à tous!!

Par ailleurs, dans ce travail, les mots énoncé et message seront employés comme synonymes. Il va sans dire que les messages sont cités tels qu'ils étaient produits par leurs émetteurs. Je n'ai changé ni le contenu, ni la mise en forme du texte des messages, ce qui fait que certaines citations apparaissent en gras, d'autres en italique etc. Parfois cependant, je me permets de mettre en relief quelques éléments afin de mieux montrer les phénomènes étudiés. Pour ce faire, je me sers du soulignement parce que ce type de mise en texte n'est utilisé dans aucune des 3000 participations.

\subsection{Choix des chats}

Les conversations de mes corpus sont tirées des trois chats suivants:

corpus I-III: Weborama (http://chat.weborama.fr)

corpus IV-X: Pause-Café (http://www.pause-cafe.net)

corpus XI-XIII: Multimania (http://chat.multimania.fr)

J'ai choisi ces chats parmi les milliers d'autres disponibles sur le net 
premièrement parce qu'ils sont francophones. Malgré la croissance de pages web rédigées dans une langue autre que l'anglais, toujours est-il que la majorité des discussions en ligne se déroulent dans la langue de Shakespeare parce qu'ils réunissent des chateurs des quatre bouts du monde. Mes trois chats par contre qui sont hébergés sur un serveur français ou canadien, ne sont pratiquement connus que dans le monde francophone. Il en résulte que les conversations se déroulent quasi exclusivement en français et que l'on a peu de risque que des non-francophones viennent s'y glisser. Ceci représente un grand avantage pour tout linguiste visant à analyser la langue française telle qu'elle est employée par les locuteurs natifs.

deuxièmement parce qu'ils sont gratuits et ouverts à tous les internautes

troisièmement parce qu'il s'agit de chats généralistes et non-modérés. Autrement dit, ils ne portent pas sur un sujet particulier et ils ne sont pas caractérisés par la présence d'une personne qui dirige la conversation et qui distribue les tours de parole (comme par exemple dans un chat avec un homme politique ou dans un séminaire virtuel proposé par une université).

et finalement parce que les conversations sont faciles à enregistrer (ce qui n'est pas le cas par exemple dans les chats basés sur le langage Java script).

\subsection{Enregistrement des conversations}

Au total, il s'agit de treize sessions dont les bornes sont complètement arbitraires. Les conversations ont été coupées dans le but d'obtenir 1000 messages d'usagers pour chacun des trois chats. En ce qui concerne le corpus du chat de Weborama ainsi que de Multimania, il se compose de trois sessions différentes. Etant donné le fait que le chat de Pause-Café est beaucoup moins fréquenté, j'ai augmenté le nombre de corpus à sept afin de recevoir un maximum de chateurs différents.

Les conversations ont été enregistrées dans des jours et des tranches horaires différents. Je suis toujours entrée dans les chats sous le pseudonyme souris, à une exception près (corpus II) où je me suis nommée jardin. Je n'ai envoyé aucun message, ni au public ni en privé. Deux participants m'ont adressé la parole. J'étais tentée de leur répondre, mais j'ai décidé de rester silencieuse afin de ne pas trop influencer la suite de la conversation.

Je n'étais pas connectée lors des conversations des corpus IV-X parce que le chat de PauseCafé offre la possibilité d'enregistrer tous les messages qui ont été affichés pendant les 24 heures précédentes. L'aspect négatif en est que cela concerne uniquement les participations des usagers, et non pas les messages automatiques du système. On ne peut donc que supposer quand exactement un utilisateur a rejoint ou quitté le salon. Les côtés positifs sont l'absence d'un "voyeur" et la possibilité de recevoir ainsi des corpus où deux personnes parlent à cœur ouvert sans être "observées". La conversation entre Petit-Lion et Maryse (corpus IV) ainsi que celle entre _Matrix_ et Lauriekkmin (corpus IX) en sont de bons exemples. Je suis bien sûr consciente du côté moral de ce procédé, mais je ne crois pas mépriser la vie privée d'autrui en enregistrant et en analysant de tels échanges. D'une part parce que, de toute façon, on a affaire à des anonymes dont on ne connaît que le pseudonyme. D'autre part parce qu'il ne s'agit pas 
d'amoureux menant une conversation chaleureuse en toute intimité mais tout simplement d'amis (virtuels) ou même d'inconnus ne parlant que de choses anodines.

\subsection{Weborama}

Weborama est un annuaire de sites web qui offre des services pour webmestres (un webmestre est une personne responsable d'un site web). Un de ces services est la mise à disposition d'un chat, appelé aussi le Webochat. Celui-ci est gratuit et ouvert à tout internaute, non seulement aux webmestres abonnés à Weborama, parce qu'à l'aide de ce service, Weborama gagne doublement: premièrement en termes de notoriété, et deuxièmement en termes de satisfaction des webmestres abonnés. On peut joindre le chat à partir de la page http://chat.weborama.fr où il faut entrer un pseudonyme quelconque. Au cas où le surnom choisi serait déjà en cours d'utilisation, l'accès au chat est refusé et on doit essayer un autre pseudonyme, par exemple souris 2 au lieu de souris. Le système n'accepte donc pas des clones (deux utilisateurs ayant le même surnom lors d'une session). C'est celui qui choisit le premier un pseudonyme qui en devient le possesseur. Il le conserve tant qu'il reste connecté et le perd dès qu'il quitte le chat. Notons de plus que ce surnom n'est pas protégé par un mot de passe, c'est-à-dire toute autre personne peut choisir le même pseudonyme si l'on n'est pas connecté en ce moment-là. Autrement dit, la Julie avec laquelle on vient d'avoir une longue conversation aujourd'hui n'est peut-être pas la même demain, d'autant moins que ce prénom est très répandu.

\subsection{Pause-Café}

Ce chat est hébergé sur un serveur canadien, et il est fort probable que la plupart des utilisateurs viennent du Canada. À part le chat, un forum de discussion est offert.

Notons quatre différences principales par rapport au chat Weborama:

$>$ le pseudonyme est protégé par un mot de passe. Quand on visite pour la première fois la page www.pause-cafe.net, il faut s'inscrire pour devenir membre de la communauté de PauseCafé. On doit fournir son pseudonyme, nom, prénom, sexe et adresse électronique. Petit détail en passant: ces indications peuvent fort bien inventées - l'adresse email mise à part. Là, on a intérêt à se tenir à la vérité puisque le mot de passe sera envoyé par courrier électronique. L'enregistrement est alors accompli et dès lors, il suffit d'entrer dans le chat en tapant son pseudonyme et le mot de passe attribué par le système. Grâce à cette inscription obligatoire qui est gratuite d'ailleurs, c'est toujours le même individu qui se cache derrière un surnom car celui-ci lui est réservé.

il est affiché l'heure à laquelle les messages ont été postés ainsi que l'heure locale du serveur dans la barre des tâches tout en bas de l'écran. Il s'agit de l'heure canadienne bien entendu.

$>$ ce chat offre le luxe d'insérer des émoticons iconiques. Ainsi, le texte c'est pas rigolo:( , envoyé par l'utilisateur souris, sera affiché automatiquement de façon suivante (les premiers 6 chiffres indiquent l'heure du postage): 22:22:34 [souris] c'est pas rigolo 
on peut en outre choisir la couleur dans laquelle seront affichés les messages et faire apparaître le texte en gras, en italique ou souligné.

\subsection{Multimania}

Multimania est un site de communauté très connu en France. Il propose à ses membres un bon nombre de services gratuits tels que le courrier électronique ou l'hébergement de pages personnelles. Pour accéder au chat, il n'est pas forcément nécessaire de s'enregistrer. Certes, on peut devenir membre gratuitement en remplissant le formulaire d'adhésion. Ainsi, le pseudonyme sera protégé par un mot de passe. Mais on peut également se connecter en tant que simple visiteur. Dans ce cas-là, on n'a pas la possibilité de choisir un surnom. Celui-ci est attribué par le système. Par exemple, si l'on est la trentième personne à se connecter de cette manière, on recevra automatiquement le pseudonyme visiteur_30. On observe que certains internautes choisissent parfois cette façon d'entrer dans le chat tout en étant membre de Multimania. Je suppose qu'ils sont trop paresseux d'entrer leur pseudonyme et leur mot de passe ou qu'ils préfèrent être complètement anonyme tout simplement. Tel le chateur visiteur_3301 (corpus XII) qui a normalement le surnom balouf (dans la ligne 118 ci-dessous, il met ce pseudonyme entre parenthèses et il est tout de suite reconnu par pulmah) ou visiteur_632 (corpus XI) qui est connu parmi les habitués sous le nom argoat ou argo:

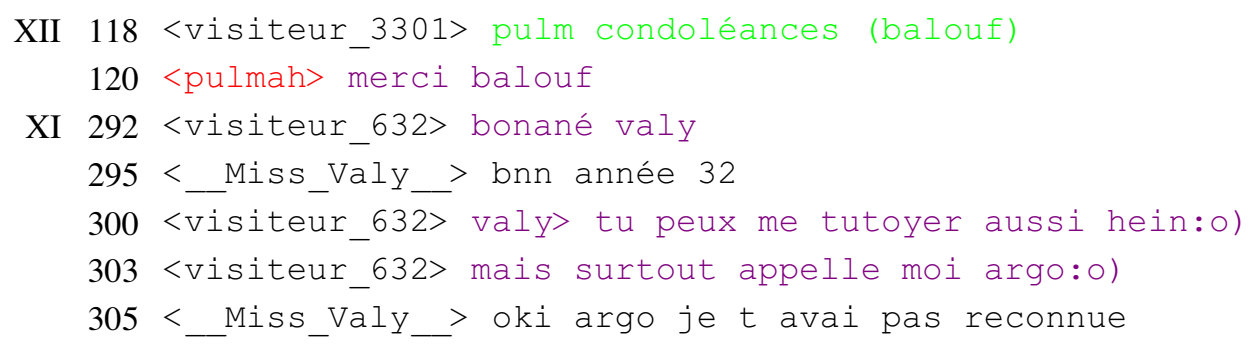

Une autre particularité de Multimania est la présence d'un opérateur. Il s'agit d'un chateur qui participe, comme tous les autres utilisateurs, à la conversation en cours, mais qui est doté de certains privilèges: au cas où un participant ne respecterait pas la chatiquette, l'opérateur peut le bannir du salon. Le participant en question sera automatiquement éjecté du chat et n'aura plus le droit d'y entrer.

Voilà une brève présentation des trois chats dont sont tirés mes corpus. Désormais, ils seront appelés Webo, Pause et Multi.

\section{$4 \quad$ Analyse des corpus}

Je commence par esquisser un inventaire des procédés de raccourcissement traditionnels présentés plus haut, à savoir les abréviations proprement dites, la troncation ainsi que la siglaison. 


\subsection{Abréviations}

\subsubsection{Abréviations proprement dites}

Quant aux abréviations par apocope, elles ne sont pas très nombreuses. Au total, j'en recueille dix. À quelques exceptions près, elles ne sont employées que par un seul chateur:

\begin{tabular}{|c|l|c|c|}
\hline abréviation & \multicolumn{1}{|c|}{ mot plein } & occurrences & $\begin{array}{c}\text { nombre de participants } \\
\text { utilisant cette abréviation }\end{array}$ \\
\hline $\mathrm{h}$ & heure & 3 & 3 \\
\hline $\mathrm{h}$ & homme & 2 & 1 \\
\hline $\mathrm{m}$ & masculin & 3 & 2 \\
\hline $\mathrm{m}$ & mètres & 2 & 2 \\
\hline $\mathrm{f}$ & féminin/femme & 8 & 7 \\
\hline $\mathrm{f}$ & francs & 1 & 1 \\
\hline $\mathrm{ff}$ & francs français & 2 & 1 \\
\hline $\mathrm{min}$ & minutes & 3 & 3 \\
\hline $\mathrm{k}$ & kilogrammes & 1 & 1 \\
\hline $\mathrm{etc}$ & et cætera & 1 & 1 \\
\hline${ }^{\circ} \mathrm{C}$ & degrés Celsius & 1 & 1 \\
\hline $\mathrm{km} / \mathrm{h}$ & kilomètres par heure & 2 & 1 \\
\hline $\mathrm{Min}$ & Minimum & 1 & 1 \\
\hline $\mathrm{Max}$ & Maximum & 1 & 1 \\
\hline
\end{tabular}

Ce qui frappe est que trois lettres représentent une abréviation pour deux mots différents: $h$ (heure/homme), $m$ (masculin/mètres) et $f$ (féminin/francs). Mais il faut dire que le contexte aide toujours à reconstituer le mot plein. Ainsi, $h$ (homme), $m$ (masculin) et $f$ (féminin/ femme) figurent exclusivement dans les messages qui répondent à une demande d'asv (âge, sexe, ville), et il est clair que le but est de désigner le sexe du participant:

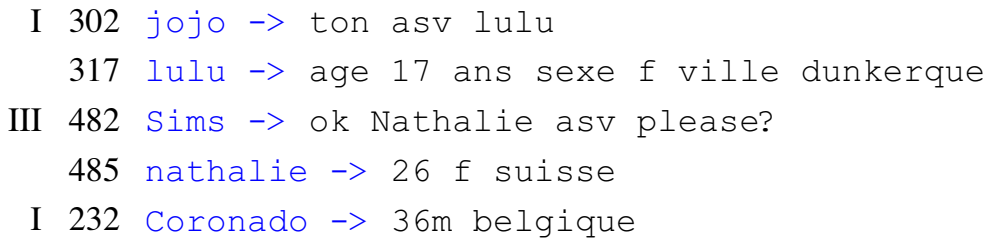

Je ne trouve ni la forme Hme (homme) ni l'abréviation Fme (femme) courantes dans les petites annonces, sans doute parce que les lettres initiales sont suffisantes. On peut même dire que les lettres $h$ (homme), $f$ (femme) et $m$ (masculin) constituent en quelque sorte des abréviations conventionnelles dans le cadre du chat.

Il est intéressant de noter que les trois dernières abréviations figurant dans le tableau cidessus, à savoir $\mathrm{km} / \mathrm{h}, \operatorname{Max}$ et Min, relèvent de l'écrit. Il s'agit de prévisions météorologiques copiées d'une page web:

IV 169 [Petit-Lion] Ce soir et cette nuit..Ennuagement ce soir suivi d'un peu de neige débutant cette nuit. Min près de moins 6 . Vents devenant du nord-est de 10 à $20 \mathrm{~km} / \mathrm{h}$ augmentant à 15 à 30 cette nuit. 
IV 171 [Petit-Lion] Dimanche..Un peu de neige cessant le matin. Dégagement par la suite. Max près de 1 . Vents du nord-est de 15 à $30 \mathrm{~km} / \mathrm{h}$ tournant à l'ouest en mi-journée.

Alors que les abréviations par apocope tiennent une place marginale, les abréviations par syncope sont plus nombreuses dans mes corpus. Près de deux tiers des utilisateurs $(65 \%)$ ont recours à une ou plusieurs abréviations de ce type. Quant aux formes, on constate une variété étonnante, mais il est à souligner que dans l'écrasante majorité des cas, il s'agit d'emplois individuels. Autrement dit, on a affaire à des abréviations où se manifeste l'usage particulier que des individus font du code graphique. Ainsi, Doc_Mabuse est le seul à supprimer la voyelle $e$ dans les articles les et des, l'abréviation $d c$ (donc) n'est utilisé que par_Beuzbeuz_, ajd (aujourd'hui) ne figure que dans un message de La_Fee_HaPercing et pts (points) est un hapax utilisé par daking:

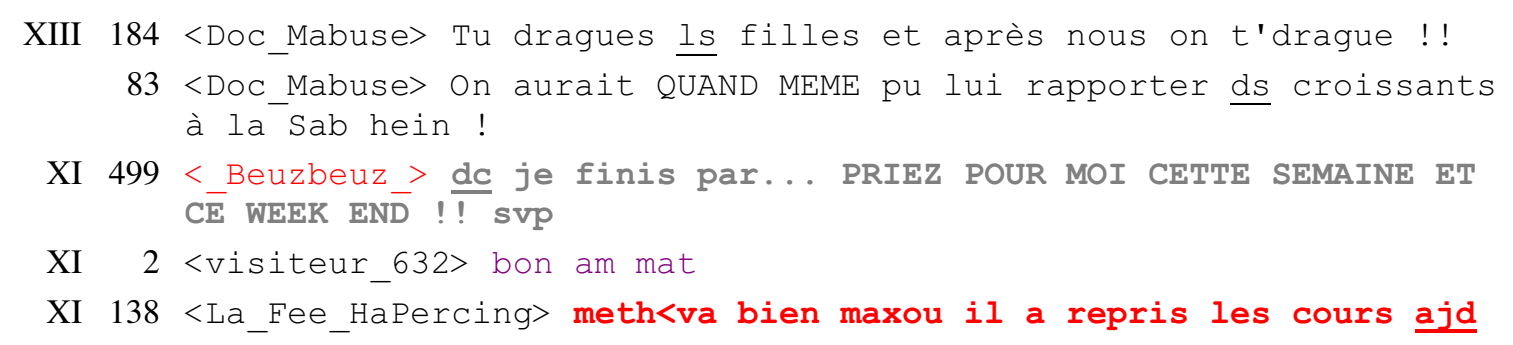

Voici les abréviations par syncope employées par au moins deux participants différents:

\begin{tabular}{|c|l|c|c|}
\hline abréviation & \multicolumn{1}{|c|}{ mot plein } & occurrences & $\begin{array}{c}\text { nombre de participants utilisant } \\
\text { cette abréviation }\end{array}$ \\
\hline slt & salut & 21 & 9 \\
\hline $\mathrm{ds}$ & dans & 7 & 5 \\
\hline $\mathrm{qd}$ & quand & 8 & 5 \\
\hline $\mathrm{tt}$ & tout & 9 & 4 \\
\hline $\mathrm{tjrs}$ & toujours & 6 & 4 \\
\hline $\mathrm{qqn}, \mathrm{qqun}, \mathrm{qq}$ & quelqu'un & 7 & 4 \\
\hline $\mathrm{pb}$ & problème & 6 & 3 \\
\hline $\mathrm{tps}$ & temps & 5 & 3 \\
\hline $\mathrm{we}, \mathrm{wk}$ & week-end & 3 & 3 \\
\hline $\mathrm{pv}$ & privé & 3 & 2 \\
\hline $\mathrm{ms}$ & mais & 10 & 2 \\
\hline $\mathrm{bcp}$ & beaucoup & 2 & 2 \\
\hline $\mathrm{mtl}$ & Montréal & 3 & 2 \\
\hline tte & toute & 3 & 2 \\
\hline $\mathrm{p}-\mathrm{etre}$ & peut-être & 2 & 2 \\
\hline $\mathrm{vla}$ & voilà & 2 & 2 \\
\hline $\mathrm{ps}$ & pas & 2 & \\
\hline
\end{tabular}

Comme le montrent ces quelques exemples, il s'agit avant tout de ramener les mots à leur "squelette consonantique" (terme emprunté à Anis 1999: 88). Ce procédé s'applique de préférence à des mots monosyllabiques, mais on trouve également un bon nombre de mots plurisyllabiques abrégés dans mes corpus. Curieusement, ces charpentes (consonantiques) ne posent pas de problèmes d'interprétation, tout au contraire, on peut les déchiffrer facilement. 
Cela tient au fait que les consonnes attribuent plus à l'identification d'un mot que les voyelles (cf. Anis 2001: 37). Les "squelettes" ci-après sont en effet largement suffisants pour l'œil (les mots pleins sont ajoutés à gauche):

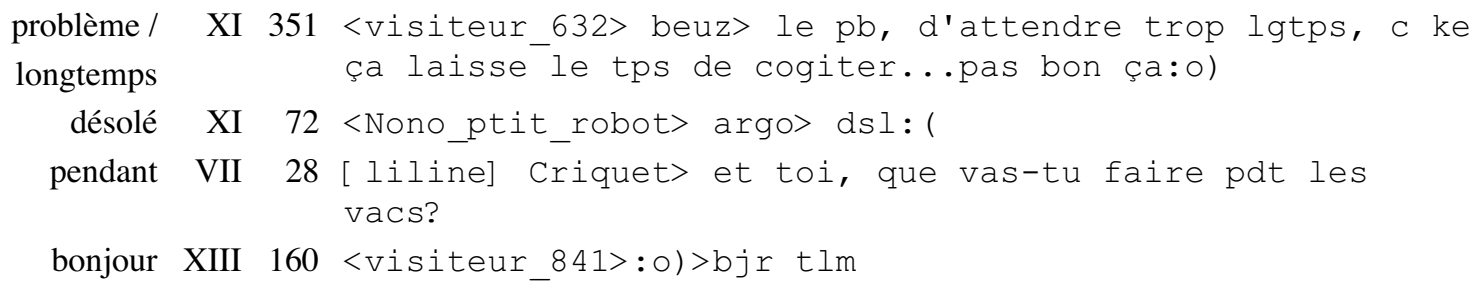

Cette facilité de reconstitution est d'autant plus étonnante que certaines formes peuvent correspondre à plusieurs lexèmes, par exemple ss (suis / sais / sans / seins / sous...) ou $t t$ (tout / tant / teint...) ou encore $v s$ (vais / vas / vous / vis / viens...). Mais dans ces cas-là, c'est surtout le contexte qui évite des interprétations erronées. Dans les extraits ci-dessous par exemple, seuls les mots vas (ligne 48), vous (ligne 327), tout (lignes 283, 47) et suis (ligne 306) sont des reconstitutions possibles:

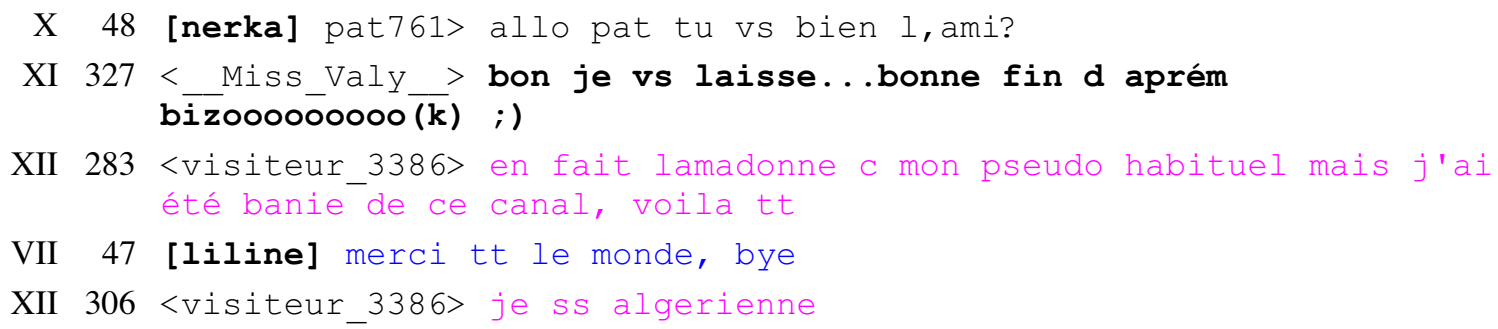

Mes corpus ne font apparaître qu'un seul exemple où un interlocuteur a des problèmes de déchiffrement:

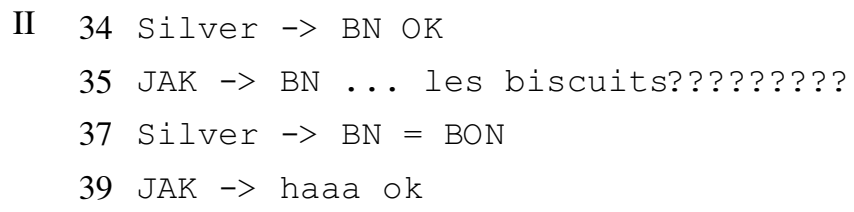

\subsubsection{Mots tronqués}

La troncation est une ressource largement utilisée dans les chats. Premièrement, elle est appliquée aux pseudonymes des participants. Comme les chateurs ont intérêt à préciser la personne à laquelle ils s'adressent pour faciliter la conversation, on trouve un grand nombre de surnoms dans les messages. Si la personne à qui l'on parle a un pseudonyme très long ou compliqué à taper, celui-ci est très fréquemment abrégé. L'échantillon ci-après par exemple montre que le surnom Akwakwak est particulièrement gênant à taper, ce qui est indiqué par les points de suspension en 174. D'où le mot tronqué $a k w$ en 178:

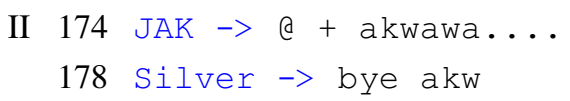


À part l'aspect pratique du raccourcissement, une motivation de la troncation est celle d'obtenir des termes d'adresse plus amicaux et conviviaux: beuz ou beuzy par exemple sont certainement plus affectueux que Beuzbeuz. Il en va de même pour sil (Silver), greg (Gregory), red (redbull), scan (Scandisk), nat (nathalie), argo (argoat), top (topstar), pul (pulmah), sem ou sema (semaphore), bal (balouf), dak (daking) etc. On pourrait en multiplier les exemples. Comme on peut le voir, dans la plupart des cas seules les premières trois ou quatre lettres du pseudonyme complet sont gardées, ce qui correspond le plus souvent à la première syllabe. On a donc affaire à des troncations par apocope.

Dans les corpus de Multi figurent également des troncations par aphérèse, étant donné le fait que ce chat offre la possibilité de se connecter en tant que visiteur. Les visiteurs se distinguent entre eux par le numéro que le système leur a attribué automatiquement lors de la connexion au salon. C'est précisément ce numéro auquel on a recours pour s'adresser à un visiteur. N'étant pas nécessaire pour l'identification, le mot visiteur est, lui, supprimé:

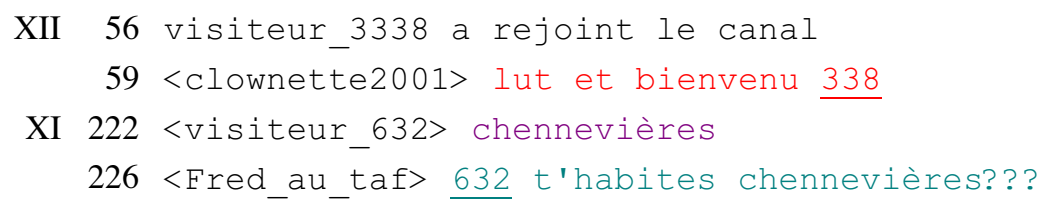

En revanche, si les nombres sont précédés d'un "vrai" pseudonyme, ils ne sont jamais mis puisqu'ils sont ressentis comme fort redondants: jop ou jo (joplaya2000), clow, clown ou clownette (clownette2001), cot ou $\operatorname{cotcot}(\cot \cot 21)$ etc.

Les troncations par syncope représentent une nouveauté dans les chats. On en trouve deux dans mes corpus:

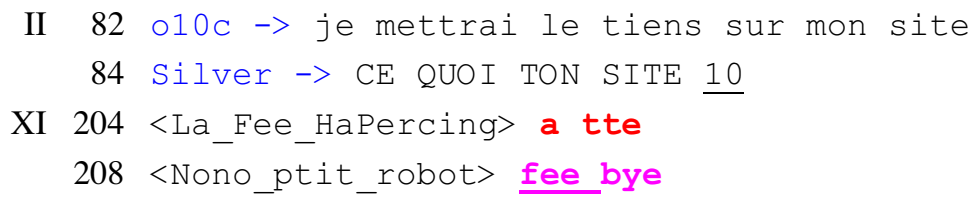

Dans les deux cas, ce sont les éléments les plus significatifs et les plus courts qui sont retenus: le chiffre 10 dans $010 c$ et le mot fee dans La_Fee_HaPercing.

Les mots tronqués sont loin de se limiter aux surnoms. Comme je l'ai déjà observé plus haut, ils n'existent, à la différence des abréviations proprement dites, pas seulement graphiquement. La stratégie de troncation est en effet très à la mode à l'heure actuelle, surtout parmi les jeunes. Les troncations envahissent le vocabulaire français, généralement en passant de l'argot à la langue familière pour figurer finalement dans les dictionnaires généraux. La langue parlée fourmille de troncats, et cette tendance se manifeste dans les chats. Ainsi, mes corpus contiennent un nombre non négligeable de mots tronqués. J'en relève 41 types différents dont les apocopes l'emportent largement. Curieusement, seulement six d'entre eux sont dignes n'un article dans le Dictionnaire universel francophone (Guillou 1999), à savoir météo (météorologique), bac (baccalauréat), métro (métropolitain), maths (mathématiques) et télé (télévision). Pourtant, à quelques exceptions près (notamment les troncats qui sont en rapport avec le chat comme par exemple webo (Weborama), multi (Multimania), op (opérateur) et la 
formule re (retour)), ils sont bien en usage dans la langue parlée, c'est-à-dire dans les échanges en face-à-face. Il en témoigne le fait qu'ils figurent majoritairement dans l'excellent Dictionnaire français-anglais des mots tronqués (2000) de Fabrice Antoine (voir la bibliographie en fin d'article). Cela montre qu'il ne s'agit pour la plupart pas de créations spontanées de certains internautes puisque les termes sont déjà attestés auparavant. Parmi les nombreux exemples de mes corpus on peut citer pseudo (pseudonyme), pro (professionnel), aprèm (après-midi), asso (association), démo (démonstration), pub (publicité), ado (adolescent), champ (champagne), fac (faculté), prof (professeur), info (information), sympa (sympathique) etc. Par contre, certaines des aphérèses de mes corpus dont je relève six, semblent bien être inventés par les chateurs. Tandis que les abréviations blème (problème), jour (bonjour), phone (téléphone) et lut (salut) se rencontrent assez souvent, les créations vec (avec) et core (encore) n'existent, à ma connaissance, pas dans la langue orale.

Il est intéressant de noter qu'un troncat peut avoir des nuances spécifiques que le mot plein ne possède pas. Ainsi, les termes ordi (ordinateur), visi (visiteur) et multi (Multimania) représentent des mots ayant une connotation extrêmement positive. En revanche, les mots pleins ordinateur, visiteur et Multimania n'ont pas cet effet. La voyelle finale $i$ qui se trouve d'ailleurs dans de nombreux mots du langage des enfants, renforce le caractère affectueux. Le même phénomène est à observer dans les exemples ci-dessous. Le mot accro (ligne 40) est beaucoup moins négatif et brutal que son mot d'origine accroché où l'idée d'une vraie dépendance (de drogues), donc d'une maladie, est encore bien présente. Dans le message 421, le terme pseudonyme perd de sa nuance de mot savant suite à l'élimination de son suffixe:

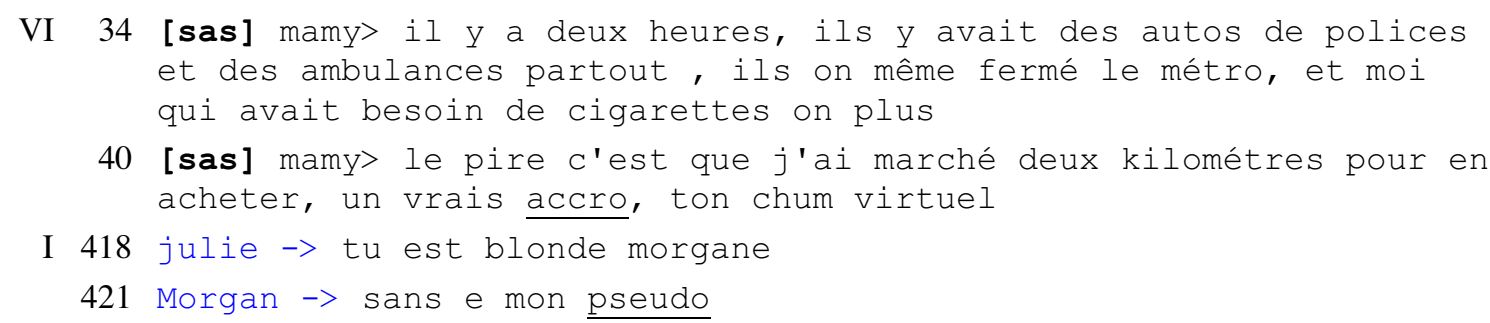

Un bon nombre des mots tronqués de mon corpus représentent des formations en - $O$ (pseudo, pro, asso, démo, ado, info...). Kilani-Schoch/Dressler qualifient le suffixe -o de "marqueur interactionnel" (Kilani-Schoch/Dressler 1999: 55). Ainsi, l'utilisation de mots tronqués crée un effet de confidentialité, de familiarité: "La suffixation en -o introduit un rapport de proximité interpersonnelle avec le ou les interlocuteurs; elle définit un espace discursif commun et exclusif au locuteur et à l'interlocuteur" (Kilani-Schoch/Dressler 1999: $55)$.

\subsubsection{Sigles et acronymes}

Dans mes corpus, la siglaison et l'acronymie se limitent à quelques expressions qui sont en revanche fréquemment utilisées. Notons tout d'abord des sigles traditionnels comme svp (s'il vous plaît, 5 occurrences) et stp (s'il te plaît, 14 occurrences). Des abréviations propres au chat sont $m d r$ (mort de rire, 50 occurrences) et lol (laughing out loud, 136 occurrences) pour manifester le rire (j'ai également compté les variantes $m d r r r$, lollll etc. dans lesquelles les 
lettres $r$ et $l$ sont multipliées pour exprimer la force du rire). Ces deux sigles sont employés de façon très inégale dans mes corpus. Sur Webo et Multi, ils jouissent d'une popularité énorme, alors que sur Pause, leur nombre est extrêmement restreint ( 3 occurrences de lol etc., 11 occurrences de $m d r$ etc.). Cela s'explique sans doute par le fait que ce dernier chat offre la possibilité d'insérer des émoticons iconiques. Ce qui est transmis sur Pause par le biais du smiley souriant ainsi que des interjections comme haha et hihi (ligne 79), est exprimé sur Webo et Multi par les sigles lol et $m d r$ :

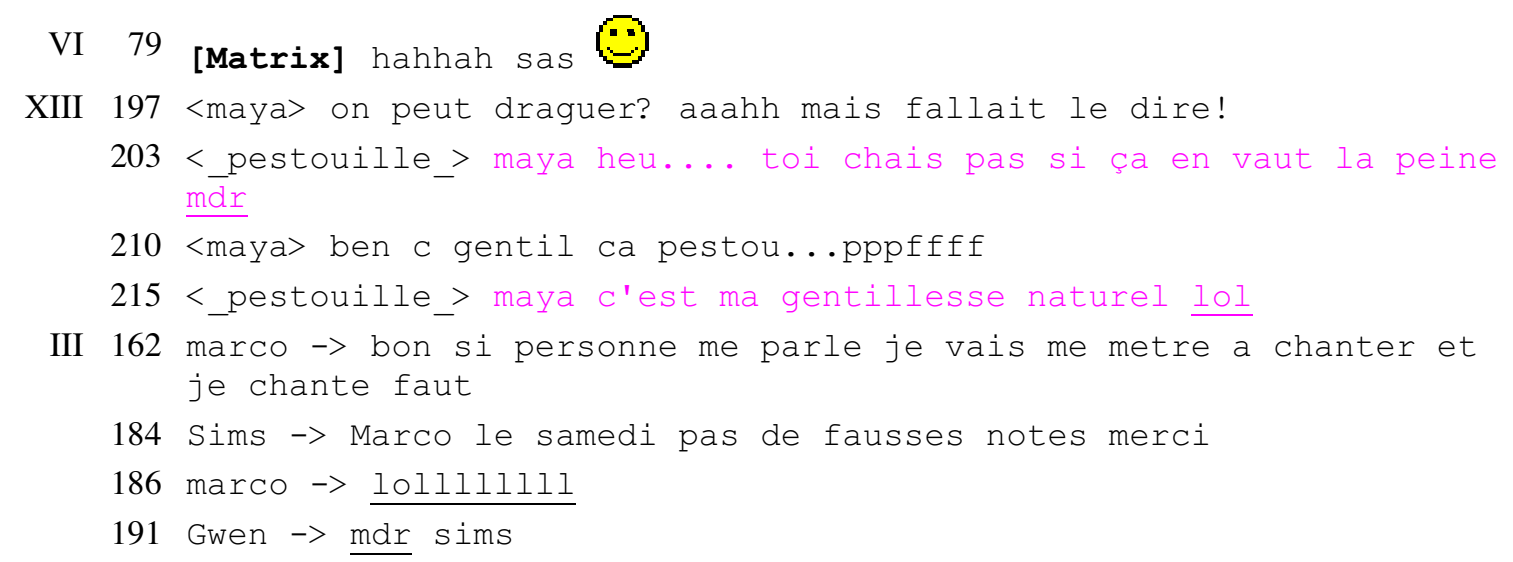

En général, les sigles $m d r$ et $l o l$ ont donc les mêmes fonctions que l'émoticon:), et ils ne sont pas intégrés à la syntaxe des messages. Cependant, les corpus font apparaître deux emplois singuliers de $m d r$, une fois en tant que verbe (ligne 134 ci-dessous), et une autre fois comme adjectif, synonyme de drôle, rigolo:

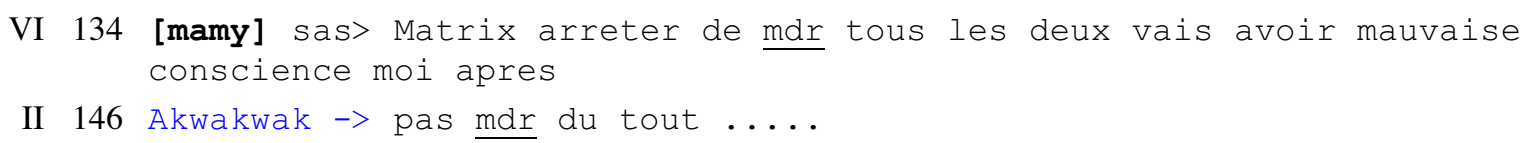

J'étais curieuse de savoir si les chateurs francophones préfèrent le sigle français $m d r$ ou s'ils ont plutôt recours à lol, son équivalent anglais, et si l'emploi de l'un ou de l'autre est conditionné par la conversation en cours. Voici les résultats de mes analyses: ${ }^{3}$

\footnotetext{
${ }^{3}$ J'ai uniquement pris en compte les conversations de Webo (I-III) et Multi (XI-XIII), vu la basse fréquence des sigles $m d r$ et $l o l$ sur Pause.
} 


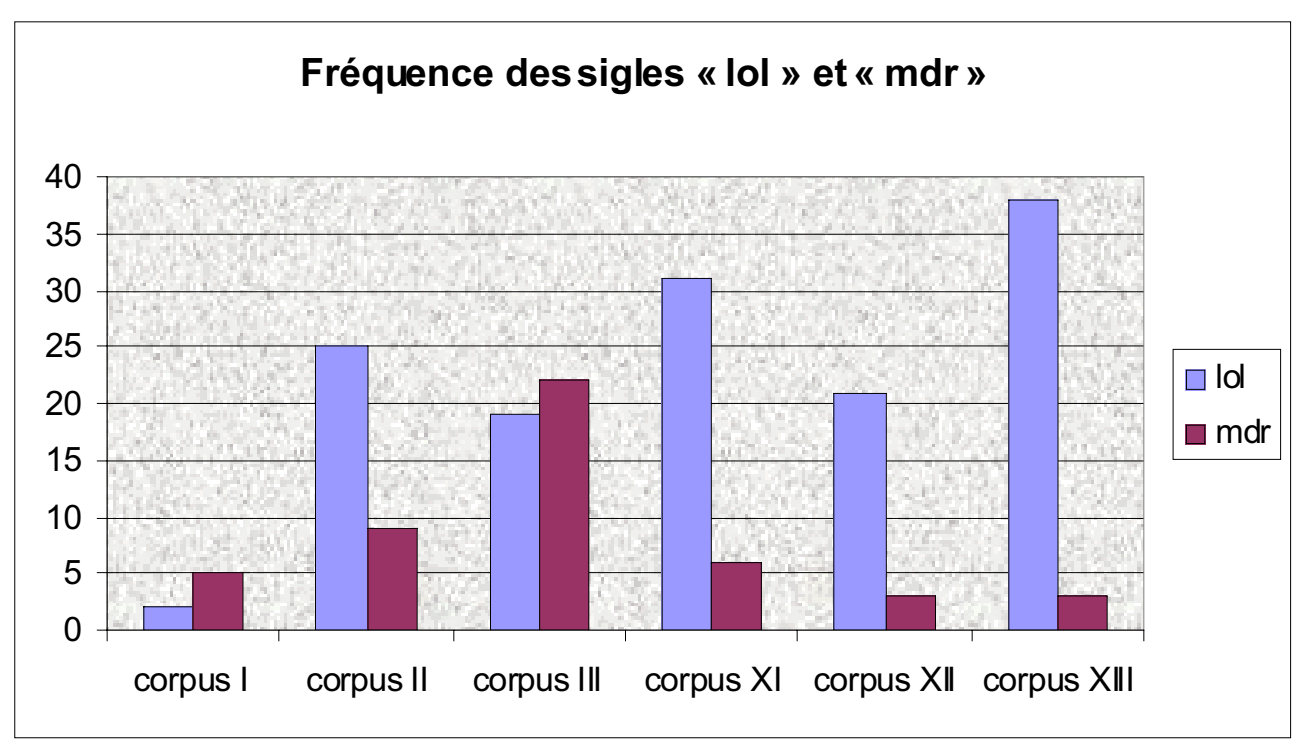

Figure 1

Le graphique (Figure 1) montre qu'à l'exception des corpus I et III, l'acronyme lol batte tous les records. Cela tient probablement au fait qu'il est beaucoup plus facile à taper: les lettres $o$ et $l$ se trouvent tout près sur le clavier (voir annexe 4) et on peut les frapper, si l'on maîtrise le système à dix doigts, avec le même doigt, à savoir l'annulaire de la main droite. Je ne crois pas que l'on ait affaire à une préférence pour l'anglais. Dans la littérature on lit souvent que les chats non-anglophones contiennent des sigles anglais tels que IMHO (In My Humble Opinion), BTW (By The Way), ROTFL (Rolling On The Floor Laughing), BRB (Be Right Back), THX (thanks) etc. (p.ex. Haase et al. 1997: 71, Wilde 2002: 15s., Bader 2002: 83, Anis 2001: 33-35). Mes corpus ne contiennent aucun de ces exemples, le sigle lol étant la seule abréviation de la langue de Shakespeare.

Un autre sigle propre aux chats et fréquemment utilisé est asv (âge, sexe, ville), une expression qui est, certes, un peu brute et laconique, mais qui permet aux chateurs de trouver le bon interlocuteur. Comme les participants ne se voient pas et ne se connaissent en général pas, on peut bien comprendre qu'ils aimeraient savoir à qui ils ont affaire. Dans l'extrait suivant par exemple, la prise de contact entre Labelle et Coronado échoue. À partir de la ligne 232, les deux Belges ne se parlent plus, sans doute faute d'affinités (enfin, Coronado a plus du double de l'âge de la fille). Sims par contre semble être bien contente de son interlocuteur JULIEN. Elle fait avancer la conversation:

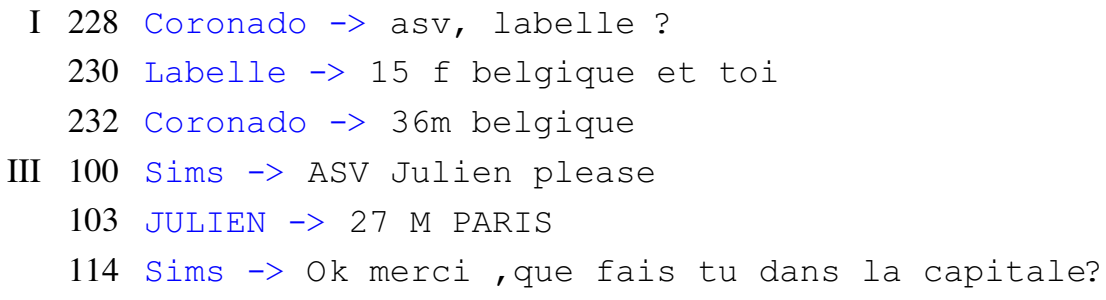

Le dernier sigle qui sera mentionné ici est tlm (tout le monde, 27 occurrences) qui est employé presque exclusivement dans les salutations d'ouverture ou de clôture pour s'adresser à l'ensemble des participants: 


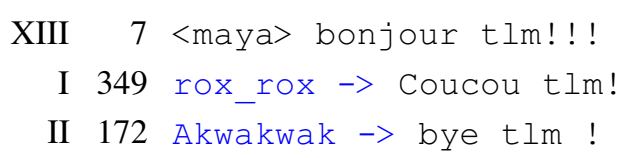

\subsection{Logogrammes}

Un tout autre procédé pour rendre plus court un énoncé consiste à utiliser des logogrammes, c'est-à-dire des "graphèmes qui correspondent directement à des morphèmes" (Anis 1999: 77). Il s'agit donc de remplacer des unités lexicales par des symboles. Ainsi, je trouve dans mes corpus les trois logogrammes + (plus), - (moins) et 1 (un). Quant au premier, il est inséré dans 24 messages envoyés par 20 utilisateurs différents. Il apparaît le plus souvent dans la formule familière à plus (plus court pour à plus tard) qui accompagne en général une salutation de clôture:

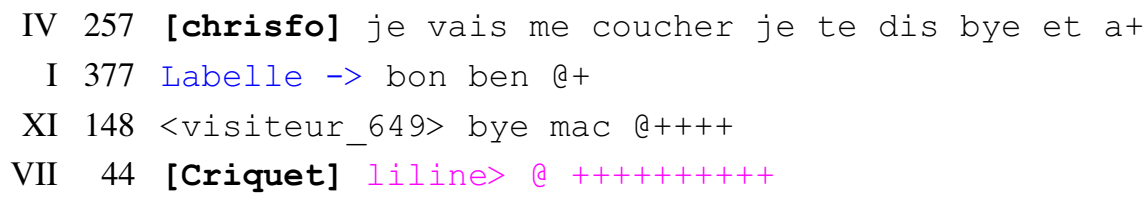

Par ailleurs, les trois derniers exemples font apparaître un usage particulier du signe arobas qui sert normalement de séparateur dans une adresse électronique. Il est utilisé dans les chats comme variante des graphèmes à et $a$, sans doute pour obtenir des effets stylistiques.

Mais retournons au signe + dont je relève d'autres emplois. Dans la ligne 502 ci-après, il fonctionne comme comparatif et dans 241 comme adverbe de négation. En 243, il fait partie de la locution en plus et en 359 finalement il transcrit le nom propre Canal Plus, une chaîne de télévision française:

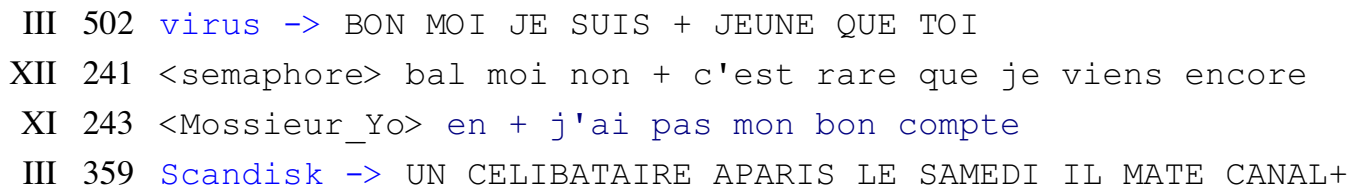

Voici un joli exemple qui me rappelle mes prises de notes dans les cours mathématiques:

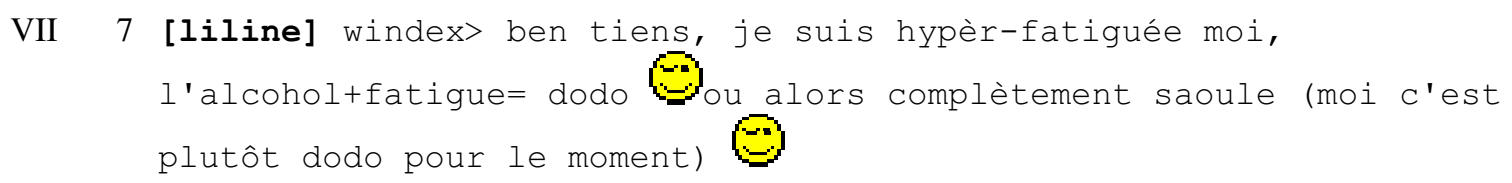

Ce message qui est particulièrement intéressant a retiré toute mon attention. Le signe d'égalité ne correspondant plus à une unité lexicale précise, on a affaire ici à une écriture entièrement dissociée de toute parole, à savoir l'écriture mathématique. L'activité de compréhension se fonde sur la seule lecture des signes. Autrement dit, l'énoncé ne sera pas oralisé par alcool plus fatigue font dodo ou quelque chose de ce genre. C'est par simple interprétation du signe plus et du signe d'égalité (et en s'appuyant sur le contexte bien entendu) que l'on peut donner 
un sens à l'équitation l'alcohol+fatigue $=$ dodo. On reconstituera par exemple Si l'on boit de l'alcool et que l'on devient fatigué, on a envie de retrouver son lit.

Le deuxième logogramme utilisé dans mes corpus est le signe - (moins), employé dans l'exemple suivant pour indiquer que les nombres ont une valeur négative. Notons qu'il s'agit d'un hapax:

VI 18 [mamy] Matrix> chez nous il y a eu -7 et dans 1 est de france -13

Le chiffre 1 pour transcrire le mot un peut également être considéré comme logogramme. Dans mes corpus, la chateuse Sims semble en être une adepte, d'autres participants ne l'utilisent qu'une seule fois:

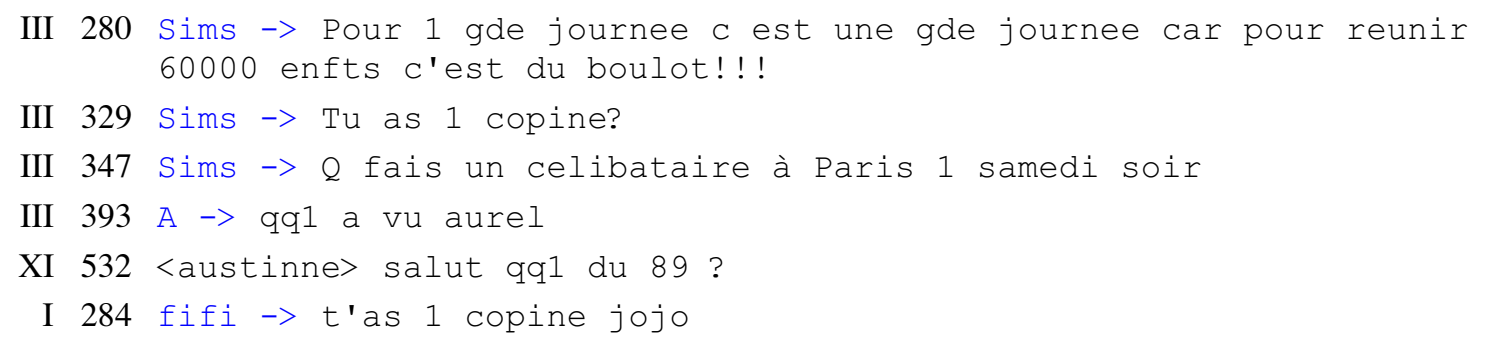

\subsection{Variantes graphiques innovatrices}

J'appelle ainsi les écarts de la norme orthographique. Le phénomène le plus évident est sans doute la réduction de $q u$ à la lettre $k$. Il s'agit d'une stratégie très répandue dans mes corpus, elle est employée par 23 participants différents.

Mes statistiques confirment la tendance observée par Anis (2001: 31) selon laquelle le procédé s'applique avant tout aux mots relatifs et interrogatifs (Figure 2). À noter que la forme kan (quand) fait en outre apparaître un autre procédé de simplification, à savoir la disparition de lettres muettes dont j'aurai l'occasion de parler plus loin.

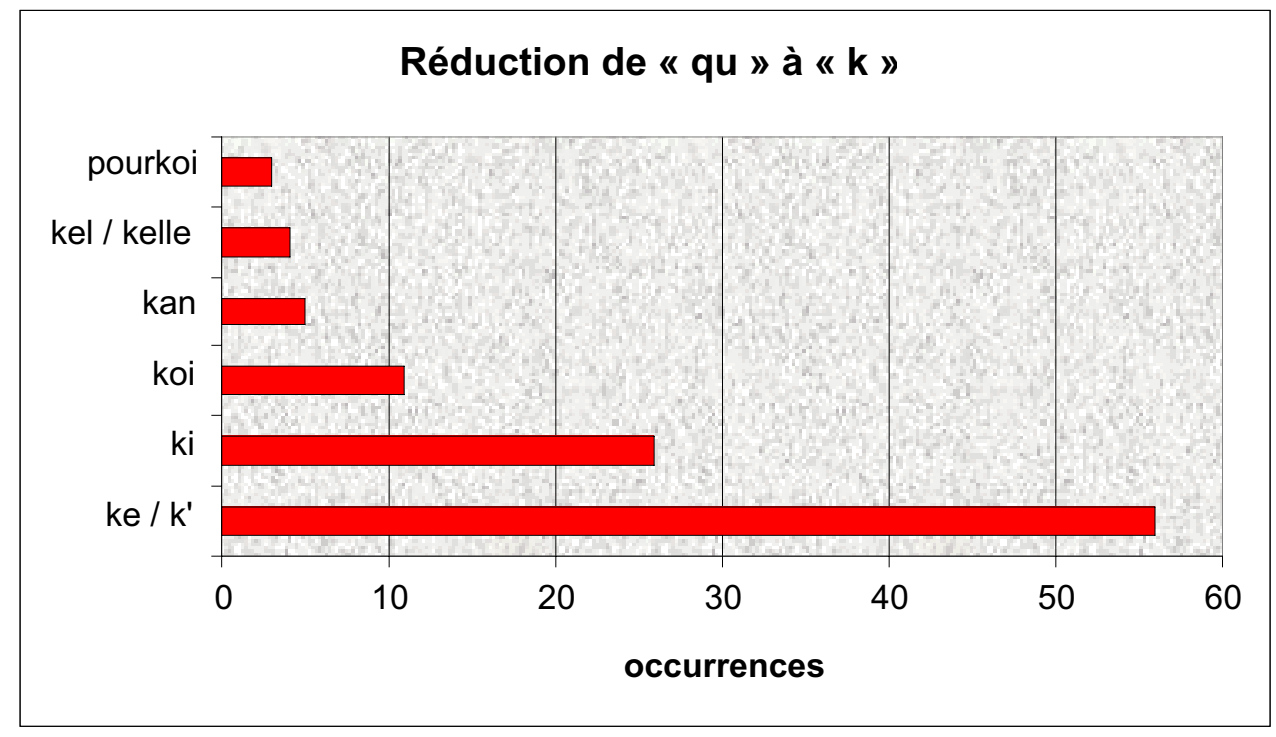

Figure 2 
Rares sont les cas où la réduction touche des mots non-grammaticaux. Je n'en trouve que quatre:

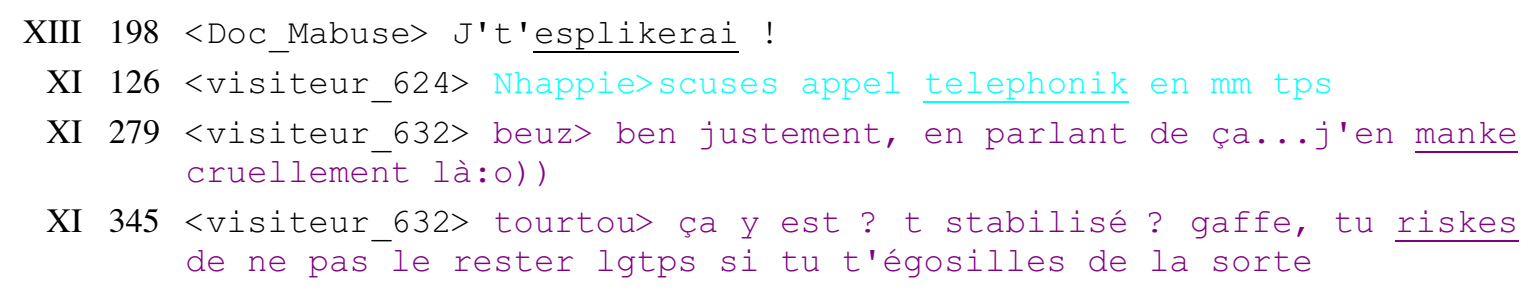

Deux utilisateurs seulement remplacent le mot que par $q$, et un participant tape $k$ au lieu de $c$ (ce qui ne présente rien d'abréviatif bien entendu, mais ce qui crée un effet stylistique):

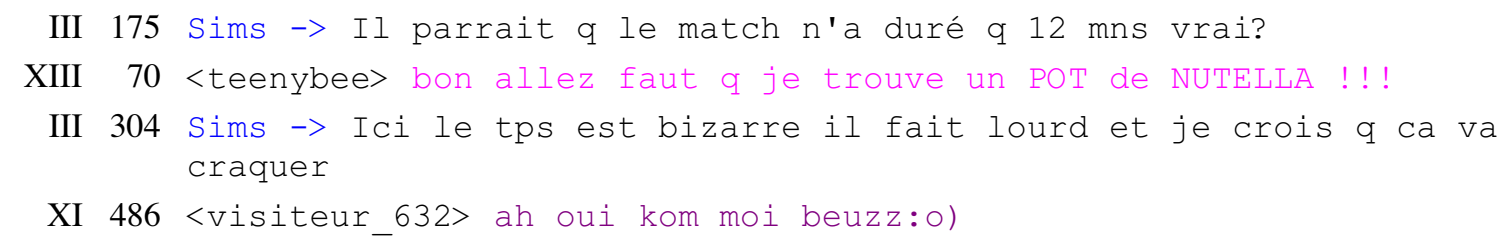

Ce dernier exemple témoigne, lui aussi, de l'omission de lettres muettes mentionnée plus haut.

Ce procédé a ses origines dans la forte redondance du code graphique. Pourquoi taper des lettres, si l'on ne les prononce pas ? - C'est sans doute ce que se demande un bon nombre de chateurs. Il n'est pas facile de savoir si les graphies suivantes sont dues à une dysorthographie de la part des émetteurs ou si elles constituent plutôt des écarts de la norme voulus. Soyons optimistes et supposons qu'elles ont été produites bien consciemment pour des raisons économiques ou ludiques (la forme "correcte" est ajoutée à gauche):

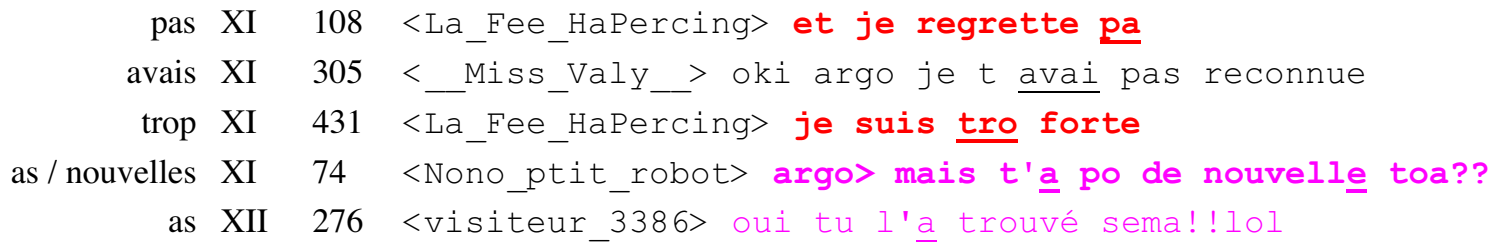

On appelle digramme un groupe de deux lettres correspondant à un phonème unique (Dubois et al. 1994: 148). Nous avons déjà vu que les chateurs mettent fin à cette faiblesse du code graphique (l'idéal graphique est qu'un phonème est représenté par un seul graphème) en remplaçant le groupe $q u$ par la lettre $k$. Le même procédé est appliqué au digramme au qui représente le phonème [o]:

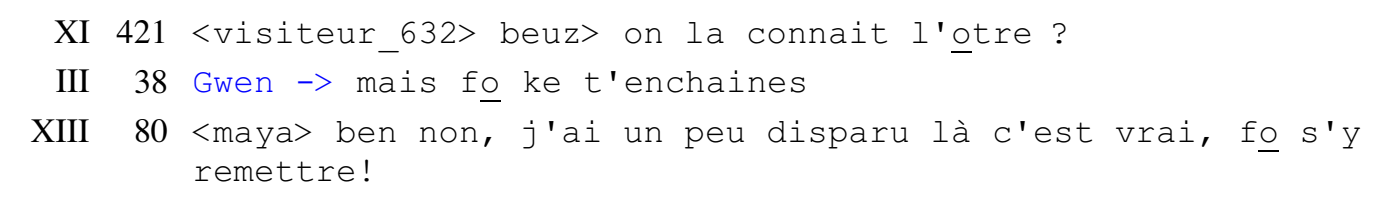

Parfois il s'agit aussi de l'o ouvert:

XIII 108 <ninjah_rastafari> cot>é bien ça ma mis de movéze humeur 
Cet exemple me conduit à mentionner une autre stratégie simplifiante qui consiste à utiliser le graphème é pour les groupes de lettres correspondant au $e$ ouvert ou au $e$ fermé. Dans les messages suivants, é remplace les graphies ait (lignes 95, 157, 466), ais (90), ez (203), et (60), ainsi que le mot es (246):

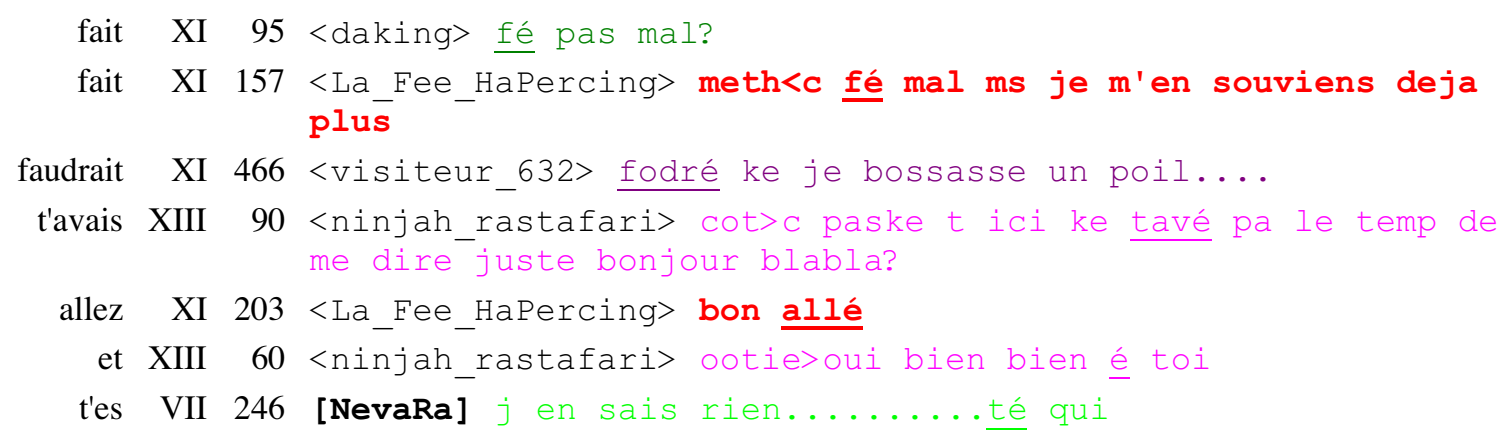

Celui qui participe pour la première fois à une discussion virtuelle est peut-être surpris voire choqué de voir s'afficher sur son écran des énoncés tels que les suivants:

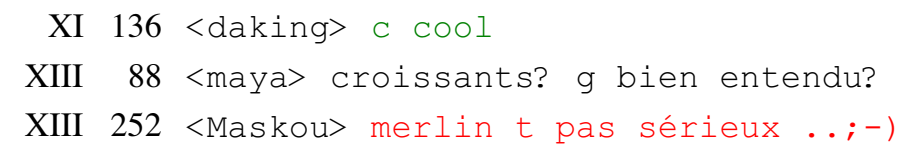

Les citations montrent que l'on profite de l'homophonie avec le nom des trois lettres $c, g$ et $t$ pour créer une graphie beaucoup plus courte de c'est, j'ai et t'es. Il s'agit de syllabogrammes (Anis 1999: 88), chaque lettre représentant une syllabe homophone. Les chateurs adorent parsemer leurs messages de telles créations remplaçant plusieurs lettres voire un ou plusieurs mots ayant la même sonorité. Elles réduisent considérablement la frappe au clavier et permettent accessoirement de passer pour un véritable initié. À souligner que le phénomène concerne surtout les mots courts et fréquents, plus précisément les verbes. En effet, mes corpus ne contiennent qu'un message dans lequel le procédé ne touche pas un verbe:

bébé XII 196 <may_> moi je dors comme un bb

Les trois syllabogrammes mentionnés plus haut sont les plus fréquents. Celui qui figure en tête de mes statistiques est $c$ (c'est) qui est employé à bout de souffle (140 occurrences, utilisé par 37 participants). La lettre $g$ (j'ai) arrive en deuxième position (15 occurrences, 7 participants) et $t$ (t'es) se hisse au troisième rang (9 occurrences, 7 participants).

On trouve parfois, mais pas très souvent, des combinaisons entre ces trois graphèmes pour mettre les verbes à l'imparfait. Tels $c t$ (c'était), $g t$ (j'étais) et $t t$ (t'étais):

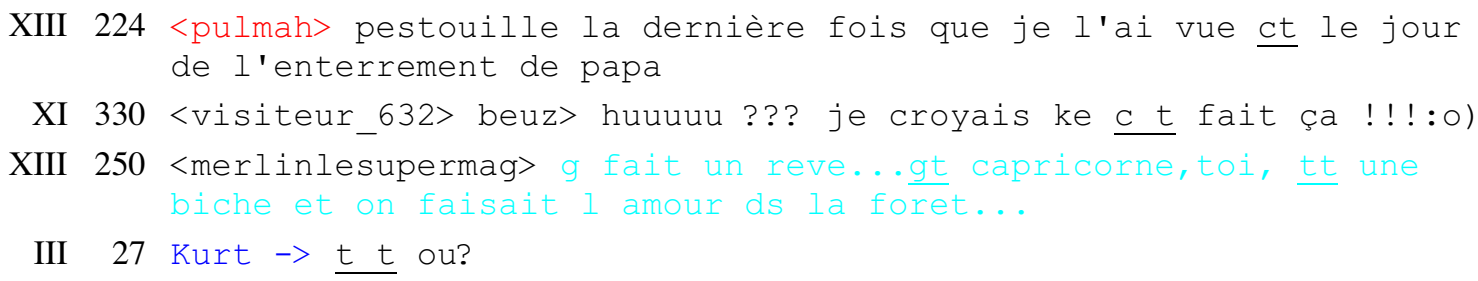


Un autre syllabogramme représente $v$ (vais). Notons que la prononciation de celui-ci, qui exige un $e$ ouvert, ne correspond plus exactement au nom de la lettre $v$ qui est [ve]. Ce syllabogramme est marginal, il ne figure que dans trois messages (dans la ligne 253, le mot à reconstituer est avait):

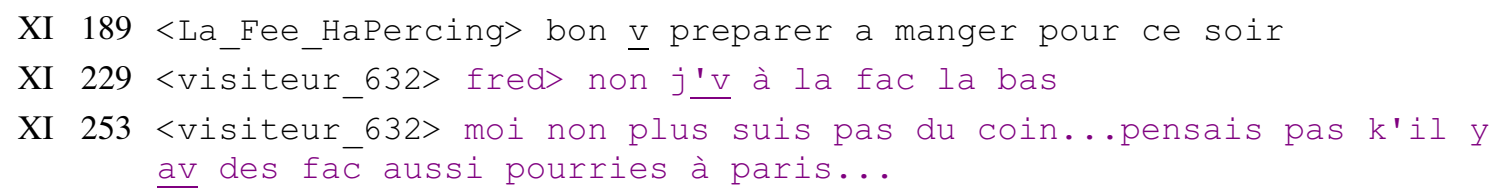

Les exemples suivants sont également très rares:

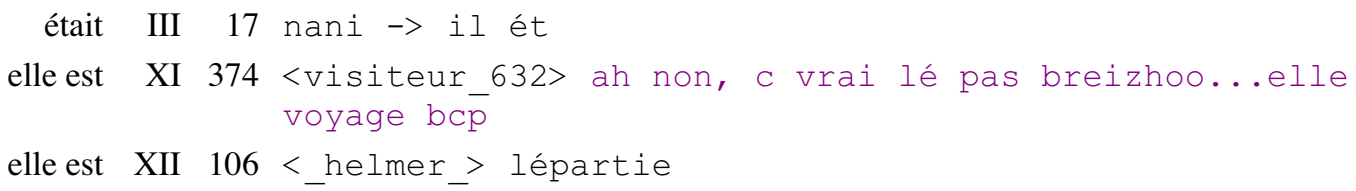

Reste la question de savoir à quel point une graphie contenant de tels éléments reste transparente. Un grand inconvénient en est en effet que la simple lecture des lettres n'est pas suffisante. On doit toujours passer par l'oralisation pour pouvoir comprendre les syllabogrammes. La chose se complique quand un seul graphème est utilisé pour plusieurs phonèmes. Dans les deux messages suivants (par exemple lignes 510 et 73), $c$ ne correspond pas à c'est (voyelle fermée), mais à sais (voyelle ouverte):

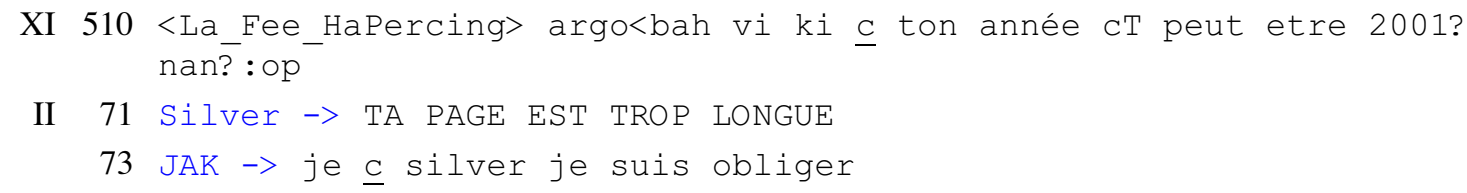

Il est important de noter que l'usage des syllabogrammes est très différent dans les trois chats. Sur Pause par exemple, seuls deux participants actifs pratiquent ce procédé alors que sur Multi, le nombre s'élève à $72 \%$ :

\begin{tabular}{|l|c|c|c|}
\hline & Webo & Pause & Multi \\
\hline $\begin{array}{l}\text { nombre des participants actifs utilisant des } \\
\text { syllabogrammes (en \%) }\end{array}$ & $12(48 \%)$ & $2(12,5 \%)$ & $18(72 \%)$ \\
\hline
\end{tabular}

Le graphique suivant (Figure 3) qui compare la fréquence du syllabogramme $c$ et celle du mot c'est dans sa graphie traditionnelle, illustre de façon encore plus prononcée les grands écarts dans les trois chats: 


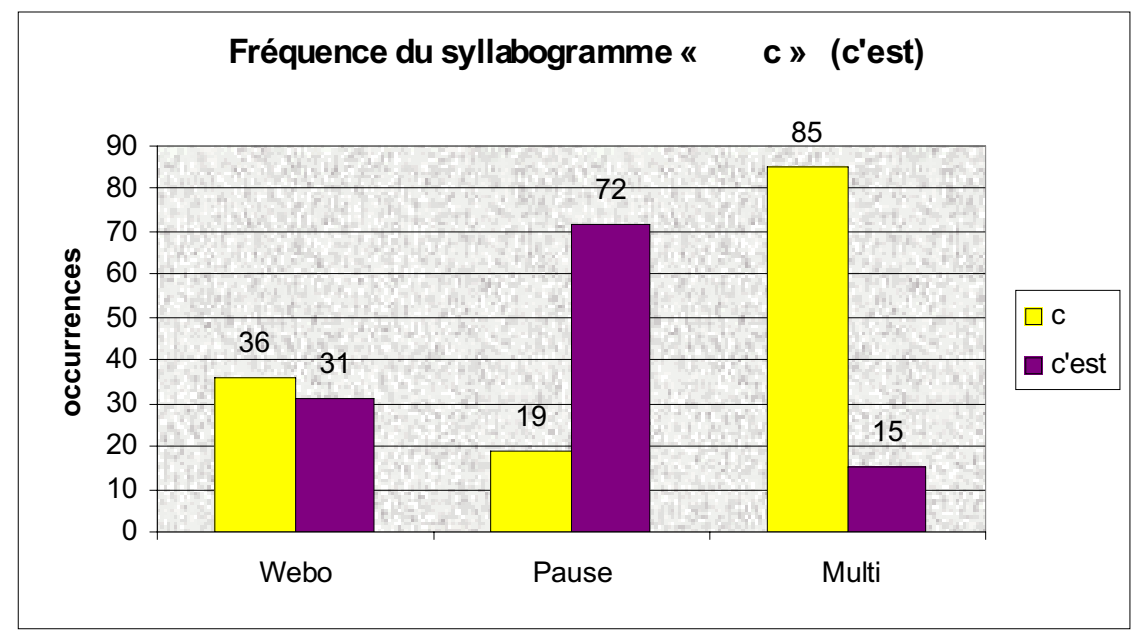

Figure 3

On voit bien que le chat de Pause-Café est celui avec le moins de syllabogrammes. Notons au passage que l'on remarque le même phénomène au niveau d'autres graphies innovatrices que je viens de présenter. Des créations telles que fot (faut) ou ki (qui) etc., tellement fréquentes sur Multi, sont largement sous-représentées sur Pause. Une explication possible s'inscrit dans la dynamique de groupe qui est certainement moins forte sur Pause où l'on a souvent affaire à des conversations entre deux ou trois personnes seulement. Les utilisateurs n'ont pas besoin de frapper, d'être original pour se distinguer des autres et pour pouvoir faire partie du groupe. Les chateurs sur Webo et particulièrement sur Multi par contre ont continûment intérêt à se faire remarquer. On a affaire à un langage de connivence, les utilisateurs cherchent à créer des effets, à coder au maximum pour être membre du groupe, pour faire partie de l'élite. De ce point de vue, la motivation des graphies innovatrices n'est donc pas l'économie, mais une certaine recherche d'originalité. En effet, mes corpus comportent un grand nombre de graphies qui ne présentent rien d'abréviatif:

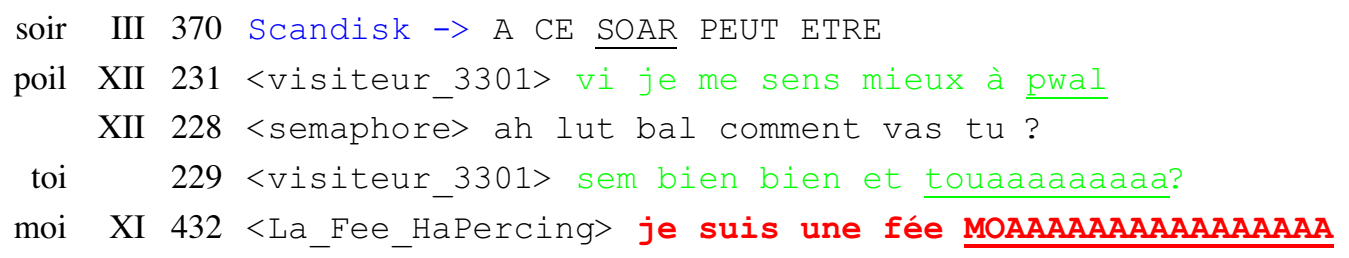

Dans ces exemples qui constituent en gros des variantes graphiques du digramme oi, c'est donc la fonction émotive qui prime, ce qui est renforcé en 229 et en 432 par l'itération de lettres.

\section{Conclusion}

Les conditions d'énonciation spécifiques de la conversation en temps réel sur l'internet ont une influence énorme sur les pratiques linguistiques et communicationnelles. Le temps presse dans les chats. Plus le nombre de participants actifs est élevé, plus le défilement des messages est vite. Ainsi, les chateurs se voient confrontés au souci suivant: comment produire un message le plus rapidement possible? Afin de résoudre ce problème, ils ont multiplié les 
stratégies de raccourcissement qui permettent de dire un maximum en un minimum de caractères ASCII. L'emploi de mots tronqués, sigles, acronymes, logogrammes et graphies innovatrices de toute sorte $(f o, c, k i$...) réduit considérablement la frappe au clavier. La plupart de ces procédés demandent en outre moins d'effort au lecteur, les nouvelles formes étant plus concises et facilitant énormément la réception. Mais cette recherche d'économie est loin de tout expliquer. On trouve aussi des graphies qui ne représentent rien d'abréviatif du tout. Si les chateurs s'écartent volontiers de la norme, c'est aussi pour impressionner, pour se faire remarquer. Plus on fait preuve d'inventivité, plus on est considéré comme "maître" de bavardage. Il s'agit d'élaborer des codes spécifiques pour initiés, et l'utilisation de ces codes contribue à l'établissement d'une sorte de familiarité et de confidentialité. Le chat créé ainsi un sentiment d'appartenance communautaire parmi ses utilisateurs, en particulier parmi ses habitués. Pour faire partie du groupe, pour pouvoir participer de façon compétente à la conversation, il faut utiliser ces codes. À ne pas oublier la dimension ludique bien entendu. "@ + tt le monde !" - Malherbe se retournerait dans sa tombe. Il est difficile de dire à quel point les nouvelles technologies et, avec elles, les nouveaux modes de communication sont susceptibles de provoquer des phénomènes d'évolution linguistique - nous verrons ce que l'avenir nous apportera.

\section{Références}

Anis, Jacques (1998): Texte et ordinateur. L'écriture réinventée? Bruxelles.

Anis, Jacques (sous la direction de) (1999): Internet, communication et langue française. Paris.

Anis, Jacques (2001): Parlez-vous texto? Guide des nouveaux langages du réseau. Paris.

Antoine, Fabrice (2000): Dictionnaire français-anglais des mots tronqués. Louvain-la-

Neuve.

Bader, Jennifer (2002): Schriftlichkeit und Mündlichkeit in der Chat-Kommunikation.

[En ligne]. Page consultée le 15 avril 2003.

Accès: http://www.mediensprache.net/networx/networx-29.pdf

Dubois, Jean et al. (1994): Dictionnaire de linguistique et des sciences du langage. Paris.

Encyclopédie Hachette (s.a.): Encyclopédie. [En ligne]. Page consultée le 30 août 2002.

Accès: http://www.encyclopedie-hachette.com/W3E

Guillou, Michel (sous la direction de) (1999): Dictionnaire universel francophone. $2^{\mathrm{e}}$ éd. Paris.

Haase, Martin et al. (1997): "Internetkommunikation und Sprachwandel". In: Weingarten, Rüdiger (éd.) (1997): Sprachwandel durch Computer. Opladen: 51-85.

Kilani-Schoch, Marianne/Dressler, Wolfgang U. (1999): "Perspective morphopragmatique sur les formations en -o du français branché". In: Mel'cuk, Igor et al. (1999): DEC. Dictionnaire explicatif et combinatoire du français contemporain. Recherches lexicosémantiques IV. Montréal: 55-66.

Naumann, Bernd (1997): "IRCs - schriftliche Sonderformen von Mehrpersonengesprächen". In: Weigand, Edda (éd.) (1997): Dialogue Analysis: Units, relations, and strategies beyond the sentence. Contributions in honour of Sorin Stati's $65^{\text {th }}$ birthday. Tübingen: 161-178. 
Office de la langue française (2002): Le grand dictionnaire terminologique. Version 1.2.2. [En ligne]. Juin 2002 (date de révision). Page consultée le 3 juin 2002. Accès: http://www.granddictionnaire.com/_fs_global_01.htm

Runkehl, Jens/Schlobinski, Peter/Siever, Torsten (1998): Sprache und Kommunikation im Internet: Überblick und Analysen. Opladen/Wiesbaden.

Schlobinski, Peter et al. (2001): Simsen. Eine Pilotstudie zu sprachlichen und kommunikativen Aspekten in der SMS-Kommunikation. [En ligne]. Page consultée le 2 janvier 2003.

Accès: http://www.websprache.net/networx/docs/networx-22.pdf

Storrer, Angelika (2001): "Getippte Gespräche oder dialogische Texte? Zur kommunikationstheoretischen Einordnung der Chat-Kommunikation". In: Lehr, Andrea et al. (éds.) (2001): Sprache im Alltag. Beiträge zu neuen Perspektiven in der Linguistik. Herbert Ernst Wiegand zum 65. Geburtstag gewidmet. Berlin: 439-465.

Werry, Christopher C. (1996): "Linguistic and Interactional Features of Internet Relay Chat". In: Herring, Susan C. (éd.) (1996): Computer-Mediated Communication. Linguistic, Social and Cross-Cultural Perspectives. Amsterdam: 47-63.

Wilde, Eva (2002): Zwischen Mündlichkeit und Schriftlichkeit: Die Chat-Kommunikation aus linguistischer Sicht. Seminararbeit. Universität Bern, Institut für Germanistik.

[En ligne]. Page consultée le 15 avril 2003.

Accès: http://www.hrz.uni-dortmund.de/ hytex/chatbib/papers/wilde.pdf 